\title{
Eye safety in operative dentistry
}

\author{
Eye safety in operative dentistry - A study in general dental practice
}

S. L. Farrier, J. N. Farrier and A. S. M. Gilmour Br Dent J 2006; 200: 218-223

\section{Aim}

In this safety conscious age all general dental practitioners should be promoting the use of eye protection. The aim of this study was to highlight the uptake of eye protection by all clinical staff and patients within the general dental practice setting, with regard to, adequate protection and its use at appropriate times.

\section{Method}

Questionnaires were sent to $200 \mathrm{GDPs}$, chosen at random, to accumulate information on the use of protection, the preferred methods of eye protection and any experience of ocular injury.

Findings

Eighty-seven per cent of GDPs wore eye protection routinely, but their choice of protection was not always adequate and not worn for all procedures. Forty-eight per cent of GDPs had experience of ocular trauma or infection which occurred during a variety of procedures; $75 \%$ of these injuries resulted from not wearing eye protection. In contrast less than half of the dental nurses and hygienists used protection routinely, particularly when cleaning contaminated instruments. However, hygienists did wear eye protection for the majority of their patient work (96\%).

Conclusion

Eye protection use by all staff and patients is currently suboptimal to conform to published guidelines. Risks encountered within the dental environment do cause harm to the unprotected eye, which can be reduced or even eliminated by improving the uptake of suitable eye protection; several choices for eye protection are available. Each individual dentist is responsible for the uptake of eye protection for all persons in their surgery environment.

\section{IN BRIEF}

- Risks encountered within the dental environment cause harm to the unprotected eyes of both staff and patients.

- Ocular injuries and infections may have serious long term effects.

- Professional bodies and the Health and Safety Executive support and advise on the need for eye protection; several choices are available.

- Eye protection by all staff and patients is currently suboptimal, especially during surface and instrument cleaning and disinfecting.

\section{COMMENT}

The use of personal protective equipment is fundamental to the safe conduct of dentistry. This welcome article draws attention to the use, or lack of use, of eye protection by clinical staff and patients in general dental practice.

The paper begins by reviewing the rationale for, and recommendations surrounding, eye protection during operative procedures. It then reports on the findings of a postal questionnaire survey of 200 general dental practitioners in South Wales, of whom 138 responded. While the usual caveats relating to response bias apply, and the fact that the dentists responded on behalf of Professionals Complementary to Dentistry, the paper contains three important facts that are worth highlighting.

Firstly, and perhaps the most alarming finding, is that almost half of the dentists responding had experience of incidents resulting in injury to the eye, and of these $59 \%$ required hospital treatment. While the timeframe over which these incidents occurred is not given, it is noteworthy that three quarters resulted from no eye protection being worn and in the remainder, the protection being worn was inadequate.

That raises the second point to be taken from this paper, namely the adequacy of protection worn. Although the majority (87\%) of general dental practitioners claimed to use eye protection routinely, in well over half the cases, dentists were wearing personal glasses, but just $28 \%$ of these had additional side shields attached. Conventional prescription glasses, without side protection, cannot be considered to provide satisfactory protection, particularly in light of fashion trends toward smaller lenses.

The third important point to be gleaned from this paper is the fact that less than half of dental nurses wear eye protection regularly and as with all staff, the proportion wearing eye protection when cleaning instruments was low.

While the study did not investigate the barriers to the use of protective eye wear, this paper serves as a useful refresher on the importance of eye protection in the clinical setting for all members of the dental team and provides some useful reminders and guides to safe working practices.

I. G. Chestnutt, Reader and Hon. Consultant in Dental Public Health, Cardiff

doi: 10.1038/sj.bdj.4813258 